\title{
EPITAFIOS HISPANOMUSULMANES DE ARJONA
}

\author{
Francisco JaVIER Aguirre SÁdABA \\ Universidad de Almería
}

\section{INTRODUCCIÓN}

El nombre de la localidad giennense de Arjona (o Arŷūna) es conocido en las fuentes árabes, principalmente las históricas y las geográficas, sobre todo por haber sido el lugar de origen de Muḥammad b. Yūsuf b. Naṣr, el fundador de la dinastía nazarí, la cual habría de mantener en tierras de la Penibética, hasta finales del siglo $\mathrm{XV}$, el último reducto del Islam andalusí. Para conmemorar el octavo centenario del nacimiento de tan ilustre arjonero ${ }^{1}$, el Excmo. Ayuntamiento de Arjona llevó a cabo la encomiable iniciativa de organizar unas jornadas culturales que se desarrollaron entre los días 28 de octubre y 3 de noviembre de 1995, como introducción al mundo hispanomusulmán en la época del sultán Ibn al-Aḥmar. Una de las actividades más sobresalientes de esas jornadas la constituyó, sin duda, la exposición de materiales de época islámica procedentes de la propia población y de otros lugares próximos, reducida por lo que se refiere al número de piezas exhibidas, pero de gran interés debido a la calidad y a la variedad de las mismas.

Entre los materiales expuestos, de alguno de los cuales tal vez haya ocasión de ocuparse en otro momento, me parece oportuno destacar aquí, por su indudable importancia para el estudio de la epigrafía andalusí, tres lápidas hispanomusulmanas originales, de los siglos X y XII, que son las únicas procedentes de Arjona conocidas hasta la fecha. De una de ellas ya teníamos referencias porque apareció recogida en la Guía histórica y descriptiva de los

\footnotetext{
${ }^{1}$ La fecha de su nacimiento en Arjona en el año 591 (= 16 de diciembre de 1194 - 6 de diciembre de 1195), el año de la batalla de Alarcos, viene dada por Ibn al-Jațīb, Al-Ihâta fi ajbār Garnāta, ed. M. 'A. 'Inān, 4 vols. El Cairo, 1073-78, II, pp. 99, n. 6, y 100; Al-Lamha albadriyya fi l-dawla al-nașriyya, ed. A. 'Ā̄sī, Beirut, 1978, pp. 48, 49; Arié, R., L'Espagne Musulmane au temps des Nasrides (1232-1492), París, 1973, p. 55, n. 2; Aguirre Sádaba, F. J., y M. C. Jiménez Mata, Introducción al Jaén islámico, Jaén, 1979, p. 242. También aparece mencionada en el texto de su epitafio, vid.: Lévi-Provençal, E., Inscriptions arabes d'Espagne, Leiden-París, 1931, p. 145, núm. 161.
} 
archivos, bibliotecas y museos arqueológicos de España (1916-1917) ${ }^{2}$ y en la Colección de R. Revilla Vielva (1924) ${ }^{3}$, y pocos años después E. Lévi-Provençal publicó el texto de la inscripción y su interpretación ${ }^{4}$. En fechas más recientes, A. Labarta la ha incluido en su estudio sobre las inscripciones árabes de Jaén ${ }^{5}$. Por este motivo, me limitaré ahora a recordar sus principales características. Se trata de una losa cuadrada de piedra caliza, cuyas dimensiones son $48 \mathrm{~cm}$ de altura $\times 53$ de largo y $16 \mathrm{~cm}$ de grosor, con un campo epigráfico de $34 \times 50 \mathrm{~cm}$ que contiene ocho líneas de texto con el epitafio de un individuo llamado 'Abd Allāh b. 'Umar al-Gāfiqī (?), fallecido un lunes de raŷab del año 549 (?) (el primer lunes de raŷab de ese año fue el día 3, correspondiente al 13 de septiembre de 1154) ${ }^{6}$. Como ya indicara E. LéviProvençal, el texto ofrece la particularidad de estar escrito en caracteres cursivos arcaizantes, incisos, con utilización de puntos diacríticos en algunas palabras. Desconozco el lugar en que se produjo su hallazgo, pero en todo caso parece que fue en el ámbito del municipio de Arjona. El original de esta lápida se guarda en buen estado de conservación en la casa de la familia Serrano, en esa población, y de ella existe una copia en yeso en el Museo Arqueológico Nacional de Madrid con el núm. 50.588 del inventario.

Las otras dos lápidas, que son el objeto del presente estudio, han permanecido inéditas, hasta donde puede saberse, y no habían sido expuestas al público con anterioridad a la celebración de las mencionadas jornadas culturales ${ }^{7}$. Me referiré a ellas con las letras A y $\mathrm{B}$, atendiendo a su antigüedad de acuerdo con la fecha que aparece en cada una de las inscripciones. La primera se encuentra actualmente depositada en una dependencia del Museo de los Santos en Arjona, habiendo pertenecido con anterioridad a un particular; ignoro su procedencia original, aunque, según las referencias obtenidas, su hallazgo debió de producirse en fecha incierta dentro del alfoz de esa localidad. La segunda lápida había permanecido, desde tiempo inmemorial, in-

\footnotetext{
2 Realizada bajo la dirección de E. Rodríguez Marín, 2 vols., Madrid, I, núm. 30.

${ }^{3}$ La colección de epígrafes y epitafios árabes del Museo Arqueológico Nacional. Madrid, 1924, p. 12, núm. 49; Patio árabe del Museo Arqueológico Nacional. Catálogo descriptivo, Madrid, 1932, p. 132, núm. 269.

4 Op. cit., p. 138, núm. 156, lám. XXXIII, c.

5 «Las lápidas árabes de la provincia de Jaén», Homenaje a Manuel Ocaña Jiménez, Córdoba, 1990, 133-134, núm. 17.

${ }^{6}$ En Lévi-Provençal, E., «D’après les tables de Wüstenfeld, le premier lundi de rağab 549 fut le 7 de ce mois, correspondant au 17 septembre 1154».

7 Debo a la amabilidad del Alcalde de Arjona, don Javier Sánchez Camacho, las fotografías de estas lápidas y la oportunidad de examinarlas «in situ». Igualmente, he de expresar mi agradecimiento al doctor V. Salvatierra Cuenca por haberme facilitado unas diapositivas de las mismas.
} 
crustada como sillar en el brocal del pozo de una casa cercana a la iglesia de San Juan Bautista, la cual se levanta entre el primer y el segundo cerco de murallas de la villa, y hoy día se conserva en casa de don José Puentes Serrano $^{8}$, vecino de Arjona.

\section{LÁPIDA A, 352 H/ 963 J.C. (LÁM. I)}

\section{a) Descripción}

Es una estela vertical de piedra caliza, rectangular, y de contornos regulares, que porta una inscripción funeraria con el epitafio de Tāhir b. 'Umar al-Rabāḥî. La fecha del óbito aparece señalada con indicación de la feria de la semana, mes y año, aunque sin mención del día, y ocurrió un martes de $\underline{d} \bar{u} l$ l-qa'$d a$ del año 352 (=21 noviembre - 20 diciembre del año 963). Esta referencia cronológica aún se puede concretar algo más acudiendo a las Tablas de M. Ocaña, de acuerdo con las cuales el primer martes del mes de $d \bar{u} l-q a^{\varsigma} d a$ de ese año fue el día 3 , que corresponde al 23 de noviembre. Se ha conservado completa y en buen estado, con escasos desperfectos apreciables que no parecen recientes, presentando sólo una zona erosionada con pérdida de materia en el lado izquierdo, que cubre las terminaciones de las líneas $2 .^{\mathrm{a}}, 3 .^{\mathrm{a}}$ y $4 .^{\mathrm{a}}$ del texto pero que no afecta a su inteligibilidad, y otra en el mismo lado, a la altura de la última línea, de la que desciende una hendidura en sentido vertical hasta la base, que parece haber borrado alguna palabra y dificulta la interpretación de la segunda mitad de esa línea.

Las medidas de la losa son $90 \mathrm{~cm}$ de altura $\times 38$ de ancho y $8 \mathrm{~cm}$ de grosor, y el campo epigráfico, de $35 \times 28 \mathrm{~cm}$, presenta un texto entero distribuido a lo largo de ocho líneas de escritura cúfica simple, incisa, cuyos caracteres se aprecian por lo general con bastante nitidez, que deja libre el tercio inferior. Carece de elementos ornamentales y está apenas desbastada, habiendo recibido el tratamiento estrictamente necesario para proceder a la inscripción del texto. Al igual que sucede en otras estelas funerarias de la provincia de Jaén que proceden de zonas rurales ${ }^{9}$, la piedra presenta en su parte inferior una forma abiselada de izquierda a derecha para facilitar su colocación.

\footnotetext{
${ }^{8}$ A quien agradezco su gentileza al pemitirme fotografiar y examinar esta inscripción en su domicilio.

9 Vid. Aguirre Sádaba, F. J., «Nuevas inscripciones árabes de la provincia de Jaén: las lápidas de Cazalilla y de Úbeda», Al-Qanțara, XVII (1996), 321-338.
} 


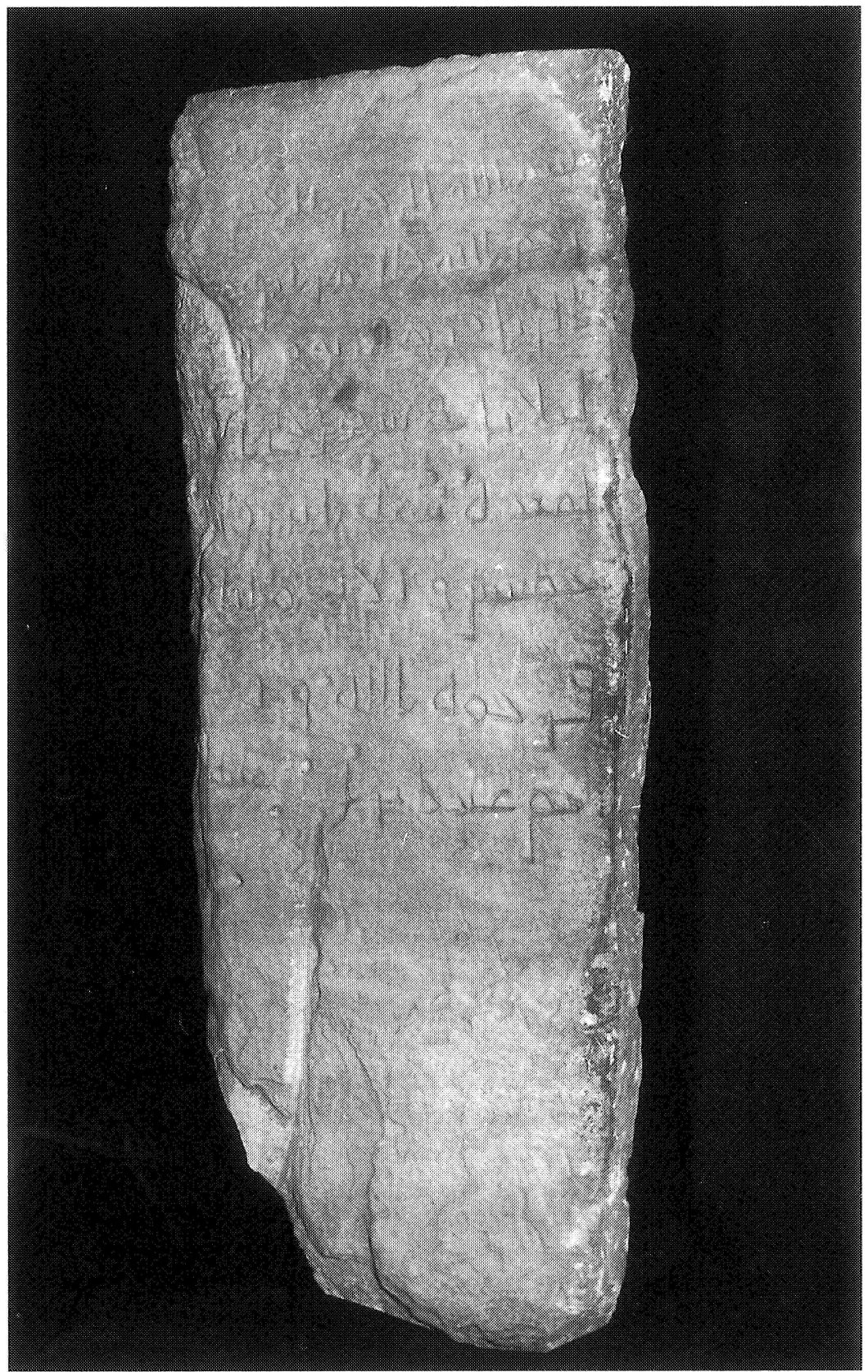

Lámina I. Epitafio de Ṭăhir b. 'Umar al-Rabāḥị. Año 352/963. 
b) Lectura

$$
\begin{aligned}
& \text { بسم الله الرحمن الرحيم } \\
& \text { رحم الله طاهر ابن (sic) عمـ[رديم } \\
& \text { الربـاحي توفي يوم الونا } \\
& \text { لثلاثا (sic) في شهر ذي الري أومي } \\
& \text { لقعدة (sic) سنة اثنن (sic) في (sic) فئاث) } \\
& \text { خمسين وثلاث مائة } \\
& \text { فرحمه الله ور } \\
& \text { حم (sic) عبده [صل (؟) (؟)...] عليه }
\end{aligned}
$$

\section{c) Traducción}

«En el nombre de Dios, Clemente y Misericordioso. / Dios se apiade de Tăhir b. 'Umar/ al-Rabāḥị. Falleció el diurno del / martes en el mes de $\underline{d} \bar{u} /$ $q a^{\varsigma} d a$ del año trescientos cincuenta y dos $(=21$ noviembre -30 diciembre del año 963. / Dios tenga piedad de él y / se apiade de su siervo, [Él] le [bendiga] (?).»

\section{d) Alifato}

A excepción de la kāf y del grupo șād-d̦ād, en esta inscripción están repesentadas todas las grafías del alifato, si bien sólo en algunas de sus posibles posiciones, además del nexo lām-alif. Por otra parte, en aquellas letras que aparecen en distintas posiciones apenas son perceptibles rasgos diferenciales, con la salvedad acostumbrada de las grafías 13f, 14f, 15f y 17f, y el caso excepcional de la 10i. El aspecto más evidente de la escritura empleada es la simplicidad de las letras, cuyos caracteres se han realizado mediante una técnica elemental de combinación de trazos rectos y curvilíneos, incisos, muy estrechos y desproporcionados entre sí, que se limitan a marcar el perfil de la grafía y le confieren una nota de rusticidad en consonancia con la apariencia general de la lápida. $\mathrm{El}$ aire estilizado que aporta a esta escritura la frecuencia y el acusado desarrollo de los trazos verticales, sobre todo en las grafías la, 1f - con una proporción media de 1 de anchura por $10 \mathrm{de}$ altura - $12 \mathrm{i}, 12 \mathrm{~m}, 14 \mathrm{f}$ y $16 \mathrm{a}$, se contrarresta por el tamaño excesivo de algunas gafías con trazos curvilíneos, también frecuentes en el tex- 


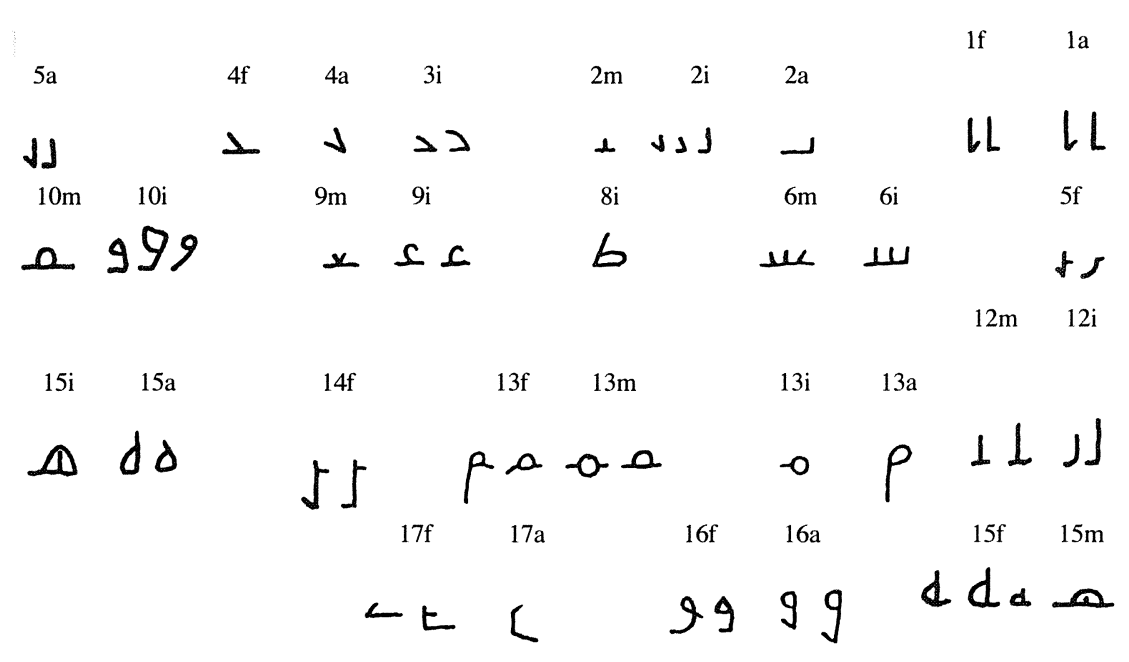

Figura I. Alifato de la lápida A.

to, como las formas $13 \mathrm{~m}, 15 \mathrm{i}, 15 \mathrm{~m}$ y $15 \mathrm{f}$. Estas observaciones se pueden verificar en el alifato adjunto elaborado para ilustrar el estudio de los caracteres gráficos de la inscripción (cf. figura I).

El alif (núm. 1), que se representa por un simple trazo vertical muy estilizado, aparece con una grafía similar en sus dos posiciones posibles, puesto que cuando va aislado incorpora un apéndice en su base, en sentido horizontal u oblicuo, igual que el trazo de la ligadura correspondiente en su posición final.

Las grafías del segundo grupo aparecen en posición inicial, medial y aislada. En el primer caso, su trazado consiste en dos cortos vástagos formando ángulo recto, salvo en la $b \bar{a}$ ' inicial de la basmala, que como suele ser habitual alcanza algo más de altura. Destaca la longitud desproporcionada del vástago de la $\underline{t} \bar{a}$ ' inicial en el numeral talăta. En otro caso (palabra $i b n)$, el trazo vertical aparece volcado hacia la izquierda. La forma medial es más baja y en la posición aislada el trazo horizontal, paralelo a la línea de escritura, duplica la longitud del vertical. Las ligaduras son generalmente horizontales, menos en la palabra $i b n$ que se hace con un trazo oblicuo.

Del grupo tercero sólo se ha utilizado la grafía de la posición inicial, representada por dos trazos rectilíneos que forman un ángulo agudo. El trazo oblicuo es más corto que el horizontal, excepto en la palabra rahmān, en la que aquél presenta cierta curvatura y se prolonga por encima de la mìm. La ligadura es horizontal. 
El grupo cuarto aparece en sus dos posiciones posibles, aislada y final, en forma de / $/$ tumbada, con el trazo superior un poco más largo que el inferior. En posición final, la ligadura es horizontal en un caso, mientras que en la palabra ' $a b d$ apenas se percibe.

Las grafías del quinto grupo se utilizan en sus dos formas, aislada y final. En la primera, es un trazo vertical que desciende más de la mitad por debajo de la línea de escritura y se dobla en un breve apéndice horizontal o sesgado. En posición final, puede sobresalir por encima de la línea y terminar en un apéndice oblicuo (como en Tâahir), o bien descender a partir de ella culminando en un apéndice horizontal.

El grupo sexto aparece en las posiciones inicial y medial, con su figura característica de /E/ volcada. En la basmala, presenta la peculiaridad de haber perdido uno de sus apéndices. Lleva ligaduras horizontales.

Del grupo octavo sólo se ha empleado la țầ' en posición inicial, cuyo cuerpo consiste en un trazo paralelo a la línea de escritura, a la cual se une por su extremo derecho mediante una curvatura y en el otro extremo termina en el asta de la letra, algo tendida hacia la derecha, que sale desde la línea y es un poco más larga que su base. El trazo de unión es sesgado.

Las grafías del grupo noveno aparecen en cuatro ocasiones, tres en posición inicial y una medial. La primera presenta forma de gancho, con una curva de mayor o menor apertura que se inicia desde la línea, alternando ligadura horizontal y oblicua, mientras que la segunda adopta forma de /V/, con ligaduras sesgadas.

El décimo grupo se presenta en dos posiciones, inicial y medial. En la primera adopta tres formas diferentes, dos de ellas con cuerpo circular o semicircular sobre base recta, trazadas por encima de la línea y encabalgadas sobre la grafía 17f, y la tercera, de tamaño desproporcionado, que consiste en un trazo vertical tan alto como un alif, con dos apéndices de distinta longitud situados en la parte superior y a media altura, que se unen cerrando el cuerpo de la letra con un trazo sesgado. La posición medial presenta una forma ovalada sobre base recta en la línea de escritura. Salvo en la partícula $f$, las ligaduras son horizontales.

La letra lām (núm. 12) se emplea en posición inicial y medial, y en ambas se reduce a un trazo vertical sin una altura uniforme en todos los casos, con la peculiaridad de que la forma medial en la palabra Allāh siempre es más corta que la inicial. Las ligaduras son de ordinario horizontales, pero en el vocablo Allāh de la primea línea la forma inicial lleva un nexo de unión curvo, y en la línea séptima, la $12 \mathrm{i}$ se une mediante trazo curvo y la $12 \mathrm{~m}$ mediante nexo oblicuo hacia arriba. 
La mìm (núm. 13) está presente en todas sus posiciones. La forma inicial y la medial consisten en un círculo, con un diámetro equivalente a la altura de $2 \mathrm{i}$, cuyo eje horizontal es la línea de escritura, aunque en rahmān y jamsin tiene un trazado más elíptico sobre base plana. La forma aislada ofrece un cuerpo ovalado por encima de la línea con un largo apéndice descendente. Esa misma figura, pero con su ligadura correspondiente, adopta la forma final, aunque en la basmala el apéndice se reduce a un breve trazo oblicuo. Las ligaduras son siempre horizontales.

La nūn final (núm. 14) es un trazo vertical que se inicia por encima de la línea de escritura y se prolonga bastante por debajo de la misma, terminando en un apéndice corto que puede ser oblicuo u horizontal.

Las grafías del grupo quince aparecen en todas sus posiciones. Las figuras aislada y final son muy similares y consisten en dos trazos en ángulo recto, el vertical de mayor longitud que el horizontal, el cual se curva hacia arriba para unirse con el anterior a los dos tercios de su altura. En posición inicial y medial, también presenta formas parecidas, adoptando en la primera un aspecto casi triangular, con un vástago vertical que surge desde la base hasta alcanzar el vértice opuesto, o algo más redondeado en la segunda, con un vástago más corto. Las ligaduras son horizontales en todos los casos.

La letra $w \bar{a} w$ (núm. 16) aparece en sus dos formas posibles, aislada y final. Cuando va aislada, la grafía presenta una cabeza semicircular y un apéndice rectilíneo descendente que puede alcanzar gran desarrollo, como en la séptima línea del texto, y se vuelve en sentido oblicuo en un tramo corto. Las dos veces que se encuentra en posición final la cabeza tiene forma triangular, pero en un caso el apéndice desciende en una curva suave y en el otro (en la palabra tuwuffiya) parece más bien una prolongación de la $t \bar{a}$ ' inicial por debajo de la línea, sin ninguna ligadura apreciable.

El grupo diecisiete se representa una vez en posición aislada y dos en posición final. En la primera, el trazado se inicia en forma sesgada por encima de la línea, para descender verticalmente y prolongarse hacia la derecha, en paralelo con la letra que le precede. En la segunda posición puede adoptar una figura similar a la anterior, con el trazo de unión correspondiente, o iniciarse en un trazo oblicuo, girando hacia la derecha horizontalmente sobre la línea.

En la inscripción se registra asimismo el empleo, por dos veces, del nexo lām-alif. En ambas ocasiones este nexo adopta la forma de lazo, con los ápices muy largos en sentido oblicuo, y aparece unido sin ningún tipo de ligadura a la grafía precedente. 


\section{e) Comentario epigráfico}

La utilización de nexos curvos de unión en la palabra Allāh, así como la altura de la traza $2 \mathrm{i}$ en la basmala, que casi duplica la de su posición medial aunque sin alcanzar la de $12 \mathrm{i}$, y la estilización en general de los trazos verticales, me inclinan a considerar la escritura de esta lápida como perteneciente al tipo cúfico simple, a pesar del uso mayoritario de ligaduras horizontales y de la angulosidad y rigidez de sus caracteres.

El texto de la inscripción presenta una estructura muy simple, adecuada a la sobriedad del mismo, a la sencillez de las grafías y a la apariencia modesta de la lápida en general. En él se distinguen las siguientes partes:

Fórmula de inauguración

Eulogia

Identidad del difunto

Fecha

Eulogia

¿Alusión al Profeta? ¿Taṣliya?

Se inicia con la basmala y continúa con una eulogia común (rahima...) que da paso al nombre del difunto, identificado mediante el ism, un nasab con un único elemento y la nisba. La expresión tuwuffiya introduce la fecha del fallecimiento, indicada con la feria de la semana, el mes y el año (sana), pero sin consignación de la data mensual, fórmula idéntica a las empleadas en las otras dos lápidas de Arjona. Se repite nuevamente la eulogia anterior y concluye con lo que puede entenderse como una alusión al Profeta seguida de tașliya, o como una eulogia de relación ${ }^{10}$, sin que el deterioro de la piedra en ese punto, que dificulta la lectura, me permita precisar más en su interpretación.

Desde el punto de vista lingüístico, en el texto se observan algunas peculiaridades que van desde el aspecto meramente ortográfico hasta el morfológico y el fonético. Por lo que se refiere a la escritura, hay que señalar la falta, corriente por otra parte en las inscripciones epigráficas, que supone la división de las palabras al pasar de línea, bien respetando las unidades silábicas, como en wa-rahima (ente las líneas séptima y octava), o sin tenerlas

${ }^{10}$ Cf. Lévi-Provençal, E., op. cit., pp. XXI-XXII; Barceló, C., «Estructura textual de los epitafios andalusíes (siglos IX-XIII)», Homenaje a Manuel Ocaña Jiménez, 45-54, especialmente 44-50. 


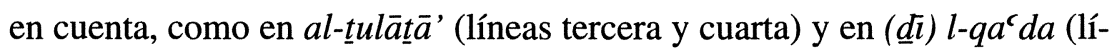
neas cuarta y quinta). Asimismo, se registra la separación incorrecta de la partícula monolítera inseparable $w a$ de la palabra que le sigue, en wa-jamsin (líneas quinta y sexta). Además, la palabra ibn (en la segunda línea) aparece precedida de alif waṣla cuando su ubicación en medio de dos nombres de persona lo hace innecesario.

Con respecto a las alteraciones morfológicas y fonéticas, hay que destacar el dialectalismo en el empleo de la forma ịtnin por el numeral cardinal ițnatayn, en contra de la norma clásica. Aquí se aprecian dos fenómenos diferentes: la omisión del morfema femenino, obviando la distinción de género, lo que es habitual en los numerales cardinales del árabe andalusí ${ }^{11}$ con el paso itnatayn>ițnayn, y a la vez la reducción del diptongo en el morfema del dual a la vocal homogénea de su semivocal (ițnayn>ițnin), en un fenómeno de metaplasmo menos frecuente, pero tampoco desconocido en el habla andalusí ${ }^{12}$ y que puede relacionarse con la expresión mi'atin (por mi'atayn) que se registra en la inscripción de Cazalilla del año 271/885 ${ }^{13}$.

El examen de la distribución del texto en el campo epigráfico delata algunos defectos que pone de manifiesto el escaso oficio del tallista. Así, se puede apreciar que los cuatro primeros renglones guardan entre sí una distancia similar, pero no mantienen la horizontalidad adecuada; en cambio los cuatro siguientes, menos torcidos, se ven excesivamente separados, debido al tamaño desproporcionado de algunas trazas, como las 15 a y $15 \mathrm{f}$ en la línea cuarta, o las que componen la eulogia fa-rahima-hu Allāh y la 16a en la séptima línea. La separación caprichosa de las palabras o de letras dentro de palabras, como en Tāhir, tuwuffiya, (di) l-qa'da ofa-rahima-hu, si bien no estorba a la interpretación del texto, más que nada por la obviedad de su lectura, permite insistir de nuevo sobre lo anteriormente expresado acerca de la deficiente calidad técnica del operario y la modestia de la lápida en su globalidad, la cual se ajusta por sus características al tipo común de epitafio de ámbito rural.

\section{f) Comentario histórico}

El desconocimiento acerca de la primitiva localización de esta losa sepulcral y de las circunstancias de su hallazgo, impide cualquier posibilidad

${ }^{11}$ Cf. Corriente, F., Árabe andalusí y lenguas romances, Madrid, 1992, p. 92.

12 Ibidem, pp. 41, 85.

${ }^{13}$ Aguirre Sádaba, F. J., «Nuevas inscripciones...», 328. 
de recurrir a otros factores, externos a la propia lápida, en un intento de ampliar el apoyo documental para su contextualización. En lo que atañe a las referencias aportadas por el contenido de la inscripción, ni el nombre del difunto, Tăhir, ni su nasab, ibn Umar, son infrecuentes en la onomástica hispanoárabe, según puede comprobarse en los diccionarios biográficos andalusíes; pero en este caso, se trata de un individuo desconocido cuya nisba, al-Rabāhịi, de acuerdo con la lectura propuesta, sugiere una relación con el topónimo de Qal'at Rabāh (Calatrava) ${ }^{14}$ cuyo carácter no puede ser precisado más allá de las simples conjeturas. Por la fecha de su muerte, en otoño del año 352/963, sabemos que vivió en la época de 'Abd al-Raḥmān III y falleció a principios del califato de al-Ḥakam II (961/976).

En cuanto a la referencia geográfica de su hallazgo, en términos genéricos la villa de Arjona y sus alrededores, hay que recordar que la primera mención acerca de esta localidad en textos árabes alude a un personaje nacido en ella, Abū Muhammad Su'ayb b. Suhayl (o Sahl) b. Su‘ayb alArŷūnī, alfaquí versado en tradiciones que estudió en Oriente y fue maestro de otro alfaquí de Arjona llamado Abū Muḥammad 'Abd Allāh b. Ibrāhīm b. Jālid, y cuya muerte tuvo lugar poco después del año $268 / 881^{15}$. Otra noticia situada casi en la misma época, en un contexto de crisis política motivada por la resistencia al proceso de estatalización emprendido por los omeyas de Córdoba, nos informa de un hecho ocurrido en el 275/888, año en el que se inicia el emirato de 'Abd Allāh, cuando el gobernador ('āmil) de la cora de Jaén, 'Ubayd Allāh b. Muhammad b. al-Gumar b. Abī 'Abda, cumpliendo las órdenes del emir, procedió a reforzar las defensas de Arŷūna y Andūŷar (Andújar) reuniendo en ambas poblaciones a los campesinos de la zona ${ }^{16}$.

${ }^{14}$ Acerca de la ciudad de Qal'at Rabāh en época musulmana, puede consultarse al-Himyarī, Kitäb al-rawd al-mi țār fí jabar al-aqțār, ed. y trad. E. Lévi-Provençal, Leiden, 1938, t. ar. p. 163, tr. p. 196; Yāqūt, Mư'ŷam al-buldān, Beirut, 1986, III, p. 23; Ibn Sa`id, Al-Mugrib fi hulà al-Magrib, ed. S. Dayf, 2 vols., El Cairo, 1978-1980, II, p. 39.

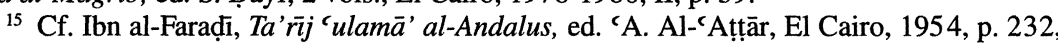
núm. 590; p. 279, núm. 734; Ibn Ḥārit al-Jusanī, Ajbār al-fuqahä' wa-l-muhadditinin, est. y ed. crít. M. L. Ávila y L. Molina, Fuentes arábico-hispanas, 3, Madrid, 1992, p. 337, núm. 473; alDabbī, Bugyat al-multamis $f i$ ta'rij riŷâl ahl al-Andalus, ed. F. Codera y J. Ribera, Madrid, 1884-1885, p. 304, núm. 843; Iyād, Tarīib al-madārik wa-taqrīb al-masâlik li-ma'rifat a' lām madhab Mālik, ed. Bukayr, Beirut, IV, p. 448; Marín, M., «Nómina de sabios de al-Andalus (93-350/711-961)», E.O.B.A., I, Madrid, 1988, 57, 123, núm. 622, y 63, 128, núm. 749.

${ }^{16} \mathrm{Cf}$. Ibn Hayyān, Kitāb al-muqtabis fi ta'rij riŷāl al-Andalus, 3. ${ }^{\mathrm{a}}$ parte, ed. M. Martínez Antuña, París, 1937, p. 51. Sobre la Arjona islámica, véase Aguirre Sádaba, F. J. y M. C. Jiménez Mata, op. cit., passim; Aguirre Sádaba, F. J., «El Jaén islámico», Historia de Jaén, Jaén, 1982, 178, 179, 190. 
LÁPIDA B, 364 H/ 975 J.C. (LÁM. II)

\section{a) Descripción}

En contraste con la verticalidad distintiva de la losa anterior, el rasgo más sobresaliente de esta lápida es su horizontalidad. Se trata de un bloque de piedra caliza, en forma de paralelepípedo y de perfiles irregulares, conteniendo una lauda funeraria a nombre de un tal Nāṣir b. Baŷila (?) [......] b. Salmūn, que murió la noche de un miércoles en el mes de $\underline{d} \bar{u} l$ l-hîŷy $a$ del año trescientos sesenta y cuatro (=12 de agosto - 9 septiembre del año 975$)$. También aquí la fecha aparece expresada con la indicación de la feria semanal, el mes y el año, pero sin especificar la data mensual; aunque se puede establecer una aproximación si tenemos en cuenta que, en ese año, el día 7 fue el primer miércoles de $\underline{d} \bar{u} l$-hîy $\hat{y} a$, correspondiente al 18 de agosto del cómputo cristiano. La lápida está completa y ofrece un estado de conservación bastante aceptable; sin embargo, además de otros desperfectos que no interesan al campo epigráfico, en la parte superior e inferior de la inscripción ha sufrido un deterioro importante con pérdida de materia. En el ángulo superior izquierdo, una mancha de cemento oculta una parte del texto e impide su lectura.

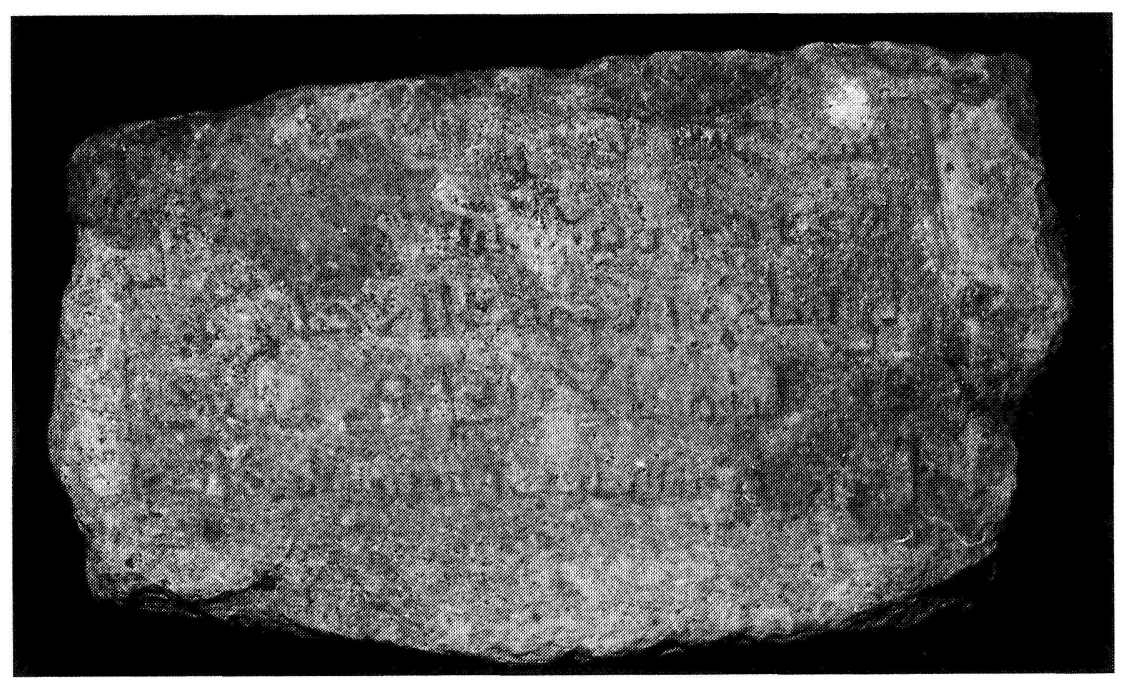

Lámina II. Epitafio de Nāșir b. Baŷila (?) [... ...] b. Salmūn. Año 364/975. 
La piedra tiene unas dimensiones de $44 \mathrm{~cm}$ de largo $\times 25$ de altura y 17 $\mathrm{cm}$ de grosor. El campo epigráfico, de $36 \times 18 \mathrm{~cm}$, muestra una inscripción entera, con cinco líneas de escritura incisa en caracteres cúficos de tipo arcaico, muy desvaídos en algunas zonas por efecto de la erosión. Se puede observar con claridad la labor de apresto de la piedra, que ha sido rebajada y enrasada en la superficie que ocupa la inscripción, de forma que el campo epigráfico queda delimitado por un marco en resalte de anchura desigual, cuya moldura ha desaparecido en su mayor parte. En una losa como ésta, de las del tipo que podemos llamar «cuadriláteros horizontales» siguiendo la denominación empleada por E. Lévi-Provençal ${ }^{17}$, la mayor longitud de los lados horizontales unida al mayor grosor de la piedra le proporcionan una base con la consistencia suficiente para mantener su estabilidad como «testigo» en la cabecera de la sepultura.

b) Lectura

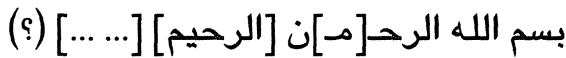

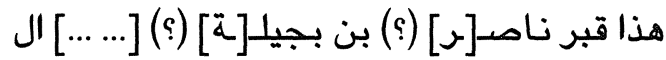

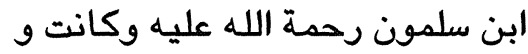

$$
\begin{aligned}
& \text { فته (sic) ليلة الاريعا في شهر ذيه الهي عليه وكاني } \\
& \text { الحجة من سنة اريعة (sic) وستين وثلث مائة }
\end{aligned}
$$

c) Traducción

«En el nombre de Dios, Clemente y Misericordioso [... ...]. ; Ésta es la sepultura de Nāșir b. Baŷila (?) [... ...] āl /b. Salmūn, la misericordia de Dios sea sobre él. Su muerte ocurrió / la noche del miércoles, en el mes de $d \bar{u} /$ / -hiŷŷa del año trescientos sesenta y cuatro (=12 agosto - 9 septiembre del año 975).»

d) Alifato

Pese al deterioro y la erosión de la piedra, podemos advertir cómo en la composición de esta lauda se han utilizado casi todas las grafías del alifato -

${ }^{17}$ Op. cit., pp. XXIII-XXIV. 


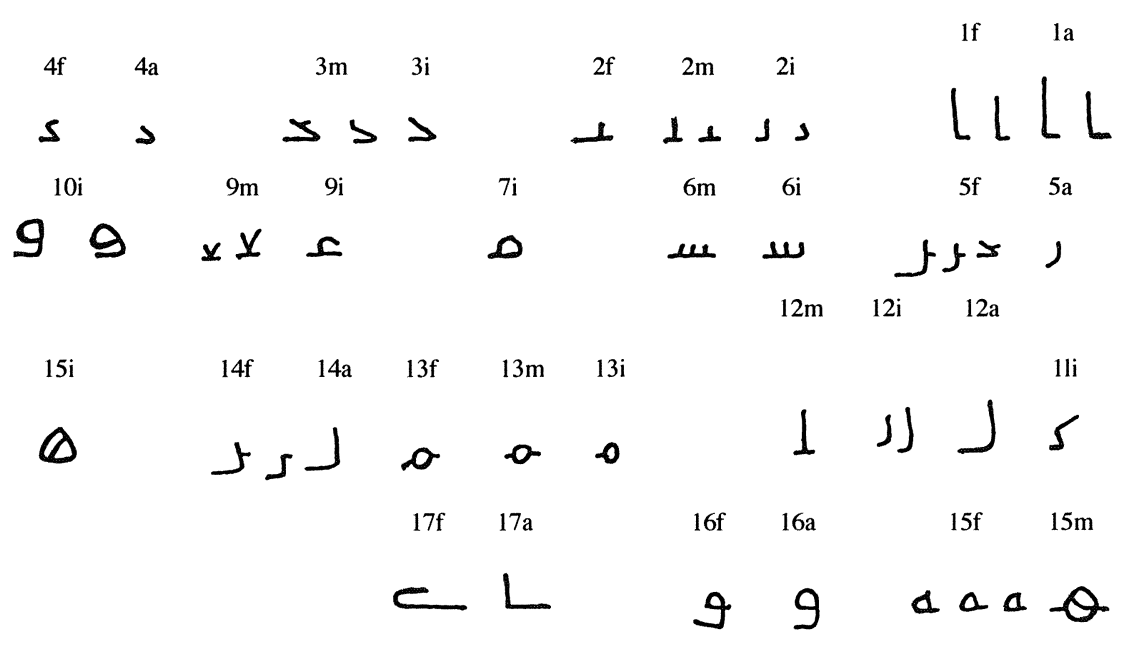

Figura II. Alifato de la lápida B.

con la salvedad del grupo $t \bar{a}^{\prime}-z \bar{a}$ - - además del nexo lām-alif, siendo digno de destacar también el empleo de las ligaduras $b \bar{a}^{\prime}-\hat{y} \vec{\imath} m, f \bar{a}{ }^{\prime}-y \bar{a}^{\prime}$ y $l \bar{a} m-h \bar{a}^{\prime}$ $\hat{y} \imath m$, con el habitual encabalgamiento de la primera letra sobre la siguiente. $\mathrm{Al}$ igual que sucede en la anterior, la simplicidad de los rasgos es la principal característica que resalta en la escritura de esta inscripción. Pero aquí los trazos son menos angulosos, y su mayor anchura unida al escaso desarrollo, en general, de los trazos verticales proporcionan a sus grafías un aspecto más recortado que contribuye a acentuar el sentido de horizontalidad de la lápida. Por otra parte, la acusada esquematización de los caracteres empleados hace que, en algún caso, apenas sea apreciable la diferencia entre grafías de distinto grupo, y, en cambio, en otras una grafía pueda presentar algunas variaciones de forma en la misma posición dentro de la palabra, como se refleja en el gráfico que recoge el alifato de esta inscripción (cf. figura II).

La letra alif (núm. 1) se encuentra en sus dos posiciones aislada y final, y lleva ligadura horizontal. En la primera posición presenta a su derecha un corto apéndice en la base. Sobresale por su altura excepcional el alif aislado de la palabra $a l-h i \hat{y} \hat{y} a$, al comienzo de la línea quinta, circunstancia que se explica por tener que adecuarse a la altura que alcanza la ligadura que le si-

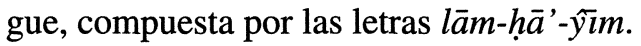

El grupo segundo se ha utilizado en sus posiciones inicial, medial y final, siempre con ligaduras horizontales. En posición inicial el cuerpo de la letra puede formar un trazo curvo antes de terminar sobre la línea de escri- 
tura, o adoptar la forma de ángulo recto lo mismo que ocurre en la posición medial y en la final. En esta última el trazo horizontal de la grafía duplica la longitud del trazo vertical; pero en la palabra kānat, al final de la línea tercera, ese trazo es más corto, obligado por la presencia inmediata de una $w \bar{a} w^{\prime}$. En el nexo $b \bar{a}$ '-ŷ̀m del nombre Baŷila la $2 \mathrm{i}$ se escribe en el punto de inicio del trazo diagonal de la $3 \mathrm{~m}$.

Las grafías del grupo tercero aparecen en posición inicial y medial, y están representadas por dos trazos rectilíneos que forman un ángulo agudo. En posición medial se encuentran dentro de las ligaduras $b \bar{a}^{\prime}-\hat{y} i m$, ya comentada, y lām-ḥa '-ŷym, en la cual el punto de unión con la grafía precedente se sitúa algo más arriba de la mitad de trazo diagonal de la letra.

El grupo cuarto está representado una vez en cada una de sus dos posiciones posibles, aislada y final. Su forma se asemeja a la grafía $3 i$ pero con los trazos más cortos. En posición aislada, al final de la línea cuarta, aparece escrita por encima de la grafía siguiente (17a) y más arriba de la línea de escritura. En posición final incorpora en su extremo superior un apéndice corto hacia la derecha. No se aprecia ninguna ligadura.

El grupo quinto se ha utilizado, asimismo, en sus formas aislada y final. En la primera está representado por un trazo curvo que se inicia por encima del renglón y desciende girando hacia la izquierda, sin alcanzar demasiado desarrollo. En posición final puede ofrecer dos grafías diferenciadas: o bien adopta la forma de la 4a uniéndose a la letra precedente en la parte baja del trazo superior, como en la palabra al-raḥmān, o bien está formada por un trazo vertical que comienza por encima de la línea y desciende curvándose luego hacia la izquierda en un apéndice horizontal que lleva ligadura rectilínea. Esta forma presenta mayor tamaño en el vocablo sahr, en la línea cuarta de texto, del mismo modo que sucede con todas las grafías que aparecen en esa línea.

El grupo sexto se encuentra en las formas inicial y medial, representado por tres ápices verticales, más bien cortos en casi todos los casos, trazados a una distancia regular entre sí sobre una línea horizontal. Lleva ligaduras rectilíneas.

De las grafías del grupo séptimo sólo se ha utilizado la șād inicial. Está formada por dos vástagos verticales de igual altura y poco desarrollo sobre una línea horizontal, que se unen por su parte superior mediante dos trazos cortos en forma de tejado. La ligadura es horizontal.

Las grafías del grupo noveno se representan en posición inicial y medial, con sus formas características de gancho abierto hacia la derecha y de $/ \mathrm{v} /$ respectivamente, y llevan ligaduras horizontales. 
El grupo décimo aparece dos veces en posición inicial con ligeras variaciones. Se representa mediante un trazo elíptico en forma de /c/ unido a otro trazo rectilíneo que puede ser vertical o estar volcado hacia la izquierda. En la partícula $f i$ de la tercera línea, la grafía se escribe montada sobre la $17 \mathrm{f}$ siguiente, por encima del renglón. La ligadura es horizontal.

La kăf (núm. 11) solamente se utiliza en posición inicial y en una ocasión. Aunque con dificultad, se puede apreciar que su grafía está formada por dos trazos en zig-zag, uno horizontal y muy corto, que es la base de la letra, y otros dos diágonales, el primero de los cuales forma ángulo hacia la izquierda con el anterior y el segundo, de mayor longitud, torna hacia la derecha.

La letra lām (núm. 12) se representa en las formas aislada, inicial y medial, y lleva ligaduras horizontales. En la forma aislada, su trazo vertical alcanza la mayor altura y en su parte inferior se curva prolongándose sobre la línea de escritura, sin descender por debajo de la misma. Esa curvatura también se percibe en la posición inicial, que puede presentar un trazo vertical más corto cuando aparece encabalgada sobre la grafía siguiente, como en al-rahmān y en al-hîŷya, sin ningún tipo de ligadura. En la palabra Allāh la forma medial es más baja.

En las tres posiciones en que aparece la letra $\overline{m i m}$ (núm. 13), inicial, medial y final, la parte inferior de cuerpo ovalado de la letra sobrepasa hacia abajo la línea de escritura. Cuando va al final, la grafía incorpora un corto apéndice por debajo del renglón. Las ligaduras salen horizontalmente de la parte central o de la mitad inferior del cuerpo de la letra.

Las grafías del grupo catorce ofrecen una notable similitud con algunas de los grupos que venimos comentando. En posición aislada adopta una forma, representada por dos trazos en ángulo recto, muy parecida a la 12a, aunque el trazo vertical es más corto y algo inclinado y el horizontal discurre un poco por debajo de la línea. En posición final, la grafía se asemeja a la $5 f$ en algunas de sus formas, pero el trazo vertical, que también aparece algo volcado hacia el lado izquierdo, no desciende tanto por debajo de renglón, y en algún caso no lo sobrepasa por arriba. Lleva ligadura horizontal.

El grupo quince se encuentra en las posiciones inicial, medial y final, y sus ligaduras son horizontales excepto en la primera posición que no lleva ninguna. Cuando es inicial tiene forma triangular, casi tan alta como un alif, con los vértices redondeados y un trazo diagonal en el interior que va aproximadamente desde el centro de la base hasta el centro del lado derecho. En posición medial presenta una grafía parecida, pero su perfil es más ovalado, descendiendo un poco por debajo de la línea de escritura y el trazo diagonal interior aparece en sentido contrario. La grafía final adopta la forma de un 
cuarto de circunferencia, o de una /c/ pegada a un vástago vertical que puede sobresalir por su parte superior.

La $w \bar{a} w$ (núm. 16) se halla representada en sus dos posiciones aislada y final. En ambas la cabeza semicircular de la letra descansa sobre el renglón y su apéndice final desciende poco por debajo del mismo, terminando en un trazo horizontal hacia el lado izquierdo.

La $y \bar{a}$ ' aislada (núm. 17) ofrece, junto con la 14a, una de las grafías más singulares de esta inscripción. De igual modo que aquélla, la 17a aparece formada por dos trazos en ángulo recto, pero aquí se abre hacia el lado derecho, su lado horizontal tiene mayor longitud y toda la letra se sitúa por encima de renglón. La grafía final se encuentra formando parte de la ligadura $f \bar{a}^{\prime}-y \bar{a}^{\prime}$, y consiste en un trazo semicircular más arriba de la línea que se prolonga sobre ésta en sentido horizontal hacia el lado derecho, paralelamente y por debajo de la letra que la precede.

El nexo lām-alif, bastante desproporcionado, presenta su habitual forma de lazo con los ápices muy abiertos en sentido diagonal.

\section{e) Comentario epigráfico}

A pesar de tener, presumiblemente, la misma procedencia y del corto espacio de tiempo - doce años- que separa la ejecución de esta lauda de la precedente, se pueden apreciar notables diferencias de factura entre ambas que apuntan a la posibilidad de atribuirlas a distintos artífices. Tanto el aspecto más pulcro de la piedra y del trazado de la inscripción, como la disposición más cuidada del texto, no obstante alguna incorrección ortográfica y en la colocación de algún vocablo que enseguida comentaré, ponen de manifiesto que el tallista, en esta ocasión, parece haberse esmerado más en su labor que en el caso anterior.

En cierto modo, no deja de ser perceptible un contraste entre esa mayor preocupación aparente por el cuidado de las formas y el severo laconismo de la inscripción que ofrece una estructura textual extremadamente simple, similar a la del epitafio de Cazalilla ${ }^{18}$, a tenor del siguiente esquema:

Fórmula de inauguración

Identidad del difunto

Fecha

Eulogia

18 Aguirre Sádaba, F. J., «Nuevas inscripciones...», 327. 
El texto comienza con la basmala como fórmula introductoria, sin que por causa de la salpicadura de cemento que cubre el final de la línea puedan reconocerse las palabras siguientes. Pero dado que el trazado de lo que podemos interpretar deja libre un tercio de línea, cabe pensar que en ese espacio se haya grabado, a continuación de la anterior, otra jaculatoria, acaso la hamdala, de la misma forma que ocurre en otras laudas contemporáneas de ésta y en la de Arjona del s. XII.

La expresión $h \bar{a} \underline{d} \bar{a} q a b r$ da paso a la identidad del difunto, enunciado por el ism y el nasab con tres elementos al menos, en lo que parece adivinarse cierto interés por resaltar su ascendencia familiar. La lectura propuesta para el nombre y el primer tramo del nasab del dedicatario de la lápida, Nāṣir b. Baŷila, es conjetural y requiere un comentario, ya que sus caracteres están muy borrosos y no se leen con claridad. Los únicos rasgos medianamente apreciables - 2i, 1f, 7i- excluyen la posibilidad de una kunya e indican que la mención del difunto se inicia por un ism del modelo $f a a^{c} i l$, y puesto que la segunda radical pertenece al grupo șâd-d̦âd, no parece descabellado interpretarlo como Nāșir. A continuación, la grafía de una nūn final delante de la palabra siguiente induce a pensar que se trata del término $i b n$ introduciendo al primer elemento del nasab. Éste sería un nombre compuesto por las grafías $2 \mathrm{i}, 3 \mathrm{~m}, 2 \mathrm{~m}, 12 \mathrm{~m}$, y un trazo vertical, de menor altura que el anterior, que podría corresponder a la grafía $15 \mathrm{f}$. Pero si bien el orden de las trazas citado parece ajustarse a uno de estos dos esquemas morfológicos posibles, fa ila o fu'ayla, utilizado tanto uno como otro en los nombres propios, las grafías correspondientes a las letras radicales reducen en la práctica, de manera concluyente, el número de variantes; debido a que, según parece y se puede verificar en las fuentes biográficas y en los repertorios onomásticos árabes, los antropónimos con esos esquemas morfológicos derivados de las raíces formadas por las grafías propuestas no son habituales. De hecho, el único que he podido localizar es Baŷila, nombre de un linaje de árabes qaḥ̣āníes al que se refiere Ibn Hazm en su obra de genealogía general titulada $\hat{Y}$ amharat ansāb al- ${ }^{\prime} a r a b^{19}$. Según este autor, la casa de los Banū Baŷila en al-Andalus se encontraba, precisamente, en la parte de Arbūna, que quizá haya de interpretarse como Arŷuna o Arjona, de acuerdo con la corrección sugerida por E. Terés ${ }^{20}$. Por estas razones, he optado por proponer la lectura Baŷila, con todas las reservas derivadas del confuso trazado que, en esa parte del

${ }^{19}$ Ed. Beirut, 1983, p. 390.

20 Vid.: «Linajes árabes en al-Andalus según la "Ŷamhara" de Ibn Ḥazm», Al-Andalus, XXII (1957), 343, núm. 48. 
texto, presentan los caracteres de la inscripción, y, evidentemente, sin que la posible coincidencia apuntada nos permita establecer conclusiones de ninguna clase acerca de la identidad de este personaje.

La eulogia que sigue a la mención del difunto, rahmat Allāh 'alay-hi, es de empleo corriente en los epitafios andalusíes y la encontramos también en la inscripción de Arjona del s. XII, sólo que allí incorpora otra eulogia de relación. La fecha del óbito viene introducida por la locución wa-kānat wafa$t u$-hu (sic), también de uso frecuente, y presenta la indicación de la feria de la semana, el mes y el año (sana), omitiendo la data mensual, en lo que constituye una característica común a las tres inscripciones de esta localidad. Hay que señalar que, tanto por lo que se refiere al esquema del epitafio como a las fórmulas utilizadas, se observa mayor similitud entre esta inscripción y la del año 549/1154 que entre ella y su coetánea del año 352/963 analizada en el apartado anterior.

Por lo que se refiere a las peculiaridades de carácter lingüístico que el texto presenta, sólo en una ocasión se aprecia la licencia en el remate incorrecto de la línea de escritura, con la separación de la palabra wa-fatu-hu (sic) entre las líneas tercera y cuarta del texto, observando la unidad silábica. Por otra parte, el numeral de las centenas presenta scriptio defectiva (talat $)$, y se ha suprimido el alif del vocablo wafāt en la línea cuarta. Pero al margen de estas alteraciones ortográficas, por lo demás bastante frecuentes, como es sabido, en la escritura de las inscripciones, el hecho más destacable en este aspecto es el uso de $a r b a^{\varsigma} a$ por $a r b a^{c}$ en la línea quinta, en contradicción con la regla clásica de concordancia y siguiendo la práctica dialectal de utilizar la forma del masculino en lugar del femenino en los numerales cardinales, como ya veíamos en la lápida $\mathrm{A}^{21}$.

Por lo que se puede apreciar, el examen de la disposición del texto revela una cierta pulcritud, según apuntaba con anterioridad, perceptible en la distribución dentro del campo epigráfico, la horizontalidad y la distancia de las líneas entre sí, la proporción de las grafías y la simetría en la separación de las palabras, haciendo la salvedad de dos excepciones señalables: el tamaño desproporcionado de algunos caracteres en la línea cuarta y la colocación atropellada de las palabras al final de la tercera línea. En esta última, el vocablo kānat se escribe prácticamente unido a la primera sílaba de la palabra siguiente. Y lo que resulta más sorprendente es que el tallista optase por la incorrección de separar la primera sílaba de la palabra wafăt, y de paso reducir el espacio para la escritura, por lo que no tuvo otro recurso que amon-

${ }^{21}$ Cf. supra, n. 11. 
tonar las grafías al término de esa línea, cuando pudo haberla trazado de una manera correcta en la línea siguiente, guardando la adecuada distancia entre las palabras. Este detalle viene a poner en evidencia, probablemente, el desconocimiento del artesano acerca de lo que estaba grabando.

Sin embargo, estos errores en la disposición y el trazado del texto que acabo de señalar, no restan mérito alguno a la ejecución general de la inscripción, que destaca sobre otros epitafios de la época procedentes de la misma área geográfica, y en particular en relación con las otras dos lápidas de Arjona, por el mayor esmero con el que ha sido realizada.

\section{f) Comentario histórico}

Habida cuenta de la coincidencia geográfica y cronológica, con una diferencia de pocos años que no introduce modificaciones relevantes de carácter histórico en el tiempo transcurrido entre ambas, es válido para esta lápida, en términos generales, lo comentado en relación con la inscripción precedente.

De los datos particulares recogidos en el epígrafe, únicamente pueden ser reconocidos con seguridad la fecha de la defunción y el último elemento del nasab. Los otros componentes del nombre, según la lectura hipotética sugerida, no son suficientes para permitir la identificación del personaje. De acuerdo con la información disponible, ni en Arjona ni en toda la Cora de Jaén se registra el apelativo familiar Ibn Salmūn. En cambio, sí lo encontramos en un área relativamente cercana, la ciudad de Córdoba, en dos individuos que vivieron en el siglo $\mathrm{x}, \mathrm{Abu \overline { }}{ }^{\top} \mathrm{A} \sqrt{1}$ H.asan $\mathrm{b}$. Salama $\mathrm{b}$. Mu'allà b. Salmūn ${ }^{22}$ y Abū 'Uțmān Sa ${ }^{\top} i d$ b. Salmūn b. Sayyid Abī-hi ${ }^{23}$, aunque no podemos saber si existió parentesco entre ellos, o alguno de ellos, y el Ibn Salmūn de esta inscripción.

Sea como fuere, lo cierto es que la evidente preocupación por destacar la genealogía del difunto a través del desarrollo del nasab, añadida a la mejor factura de esta lápida, que le confiere una categoría superior a los otros dos epitafios referidos, parecen indicar que, en este caso, el destinatario de la

${ }^{22}$ Tradicionista cordobés que viajó a Oriente y murió en su ciudad natal en $₫ a w w a \bar{l}$ del año 335/ mayo del 947. Vid.: Ibn al-Farađ̣ī, op. cit., p. 130, núm. 342; Ibn Ḥārit al-Jušanī, op. cit., pp. 72-73, núm. 71.

${ }^{23}$ Maestro cordobés fallecido en ŷumādà $I$ del año 380/ agosto del 990. Vid.: Ibn alFarađ̣̄i, op. cit., p. 207, núm. 526; Ávila, M. L., La sociedad hispano-musulmana al final del Califato (aproximación a un estudio demográfico), Madrid, 1985, p. 166, núm. 963. 
lauda debió de tratarse de una persona que gozara de algún relieve social o económico en su comunidad.

\section{RESUMEN}

Este artículo ofrece la transcripción, la traducción y el estudio de dos nuevas inscripciones funerarias inéditas, halladas en Arjona (provincia de Jaén) y que están fechadas en los años 352/963 y 364/975 respectivamente. La técnica epigráfica de estas lápidas es la habitual en las inscripciones de procedencia rural.

\section{ABSTRACT}

This article offers a transcription, interpretation and study of two new and unpublished funeral inscriptions, found in Arjona (province of Jaen) and dated to the years 352/963 and 364/975 respectively. The epigraphic technique for these tombstones is the usual one used in inscriptions of rural provenance. 